\title{
Phase-Field Model of Finite Interface Dissipation: A Novel Way to Directly Couple with CALPHAD Databases
}

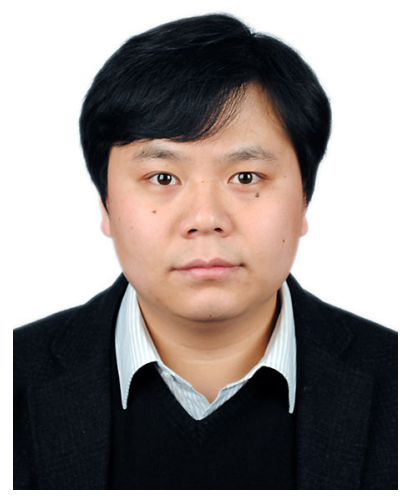

Lijun Zhang

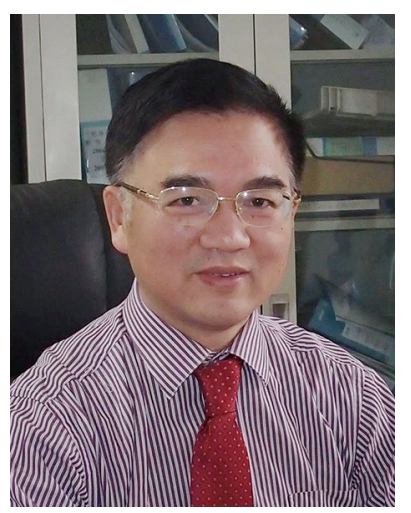

Yong Du
The phase-field method, being based on a diffuse representation of the phase boundaries, has emerged as a powerful tool to simulate the microstructural evolution in various materials processes during their life time. In order for a further quantitative simulation, three key points need to be fulfilled in general, i.e., (i) a quantitative phase-field model, (ii) input of the reliable thermophysical parameters, and (iii) key experimental validation. Nowadays, a quantitative phase-field model is usually available in the literature, especially after the work by Alain Karma from Northeastern University. ${ }^{[1]}$ In terms of key experimental validation for the simulated microstructure, a combination of classic (i.e., X-ray diffraction: XRD, metallography, Scanning Electron Microscope: SEM, Electron Probe Micro Analysis: EPMA) and modern (i.e., Electron Backscattered Diffraction: EBSD, Transmission Electron Microscope: TEM, Three-dimensional Atom Probe: 3DAP) techniques can meet the demands. While for input of the reliable thermophysical parameters, it is still a challenge for the phase-field community even today.

The thermophysical parameters needed in the phase-field simulations include bulk free energy and its derivatives (viz. diffusion potentials and thermodynamic factors), diffusion coefficients, interfacial energy, interfacial mobility, lattice parameters, elastic constants, viscosities, etc. Through the past forty-four years' development, the CALPHAD (CALculation of PHAse Diagrams) technique has been moving toward maturity. Today, with the CALPHAD technique, databases for thermodynamics, atomic mobility, lattice parameter, elastic constant, viscosity, thermal conductivity in different materials can be established, from which the respective thermophysical quantity at any composition, temperature and pressure can be retrieved. Thus, the coupling of phase-field model to the CALPHAD databases has become a good standard for dynamically providing the reasonable thermophysical information during the phase-field simulation. ${ }^{[2,3]}$

In CALPHAD-type databases, the parameters for each thermophysical property are stored phase by phase in accordance with a certain rule. Therefore, the coupling of the phase-field model to CALPHAD databases can be in a straightforward way, for instance, that the complete description for each property in each phase is directly incorporated into the phase-field model. It is true for all the above-mentioned thermophysical properties except the thermodynamic information. That is because either the condition of a giving partitioning (i.e., local equilibrium hypothesis) or the condition of equal diffusion potentials (i.e., quasi equilibrium hypothesis) is needed in traditional phase-field models (especially for the traditional coarse-grained phasefield models) for retrieving the phase concentrations and potential over the diffuse interface, resulting in complex thermodynamic calculations. Hence, the traditional coupling approaches include (i) the link to a data file containing the necessary information on free energy, chemical potentials and phase equilibria calculated by an external CALPHAD software package, (ii) the approximation of Gibbs energy curve/surface due to the CALPHAD calculations via some simple functions, and (iii) the use of locally linearized phase diagrams by running a CALPHAD software package in parallel to the phase-field simulation via an interface, e.g. TQ interface for MICRESS (MICRostructure Evolution Simulation Software). The three traditional coupling approaches are categorized as the "indirect" method, and have some inherent drawbacks. For instance, the first and second coupling approaches are very difficult to be used in multicomponent alloys, while the dynamic interaction between phase-field code and CALPHAD software package in the third coupling approach is still a time-consuming process. Moreover, most of the CALPHAD software packages are commercial and costly.

One exception in the traditional phase-field models is the treatment of $\gamma / \gamma^{\prime}$ solid state transformation in, e.g. Ni-based superalloys, during phase-field simulation using the microscopic phase-field model. ${ }^{[4]}$ For the $\gamma / \gamma^{\prime}$ order-disorder transition described by the four-sublattice (4SL) model in CALPHAD formalism, the element site fraction in 4SL model can be converted into an order parameter (i.e., a kind of phase-field variable) in the microscopic phase-field model. Thus, the Gibbs energy description for $\gamma / \gamma^{\prime}$ phases can be directly incorporated into the phase-field model. This kind of coupling approach can not only be applicable in multicomponent alloys, but also avoid the complex equilibrium calculations and energy minimization during the simulation. Unfortunately, this coupling approach is only limited up to now to the $\gamma / \gamma^{\prime}$ order-disorder transition. 
Very recently, a phase-field model with finite interface dissipation has been developed, and can provide the description of various kinetic processes at the mesoscopic scale without restriction to the type of transformation ranging from the chemical equilibrium to strongly non-equilibrium ones. ${ }^{[5,6]}$ The novel feature of this model is that each phase concentration is assigned by a kinetic equation to account for finite interface dissipation instead of applying an extra condition for solute partitioning between the phases as in traditional models. With such a novel feature, the external equilibrium calculations for the partitioning at the interface can be avoided in the phase-field simulations. For the substitutional and interstitial solution phases, their complete Gibbs energy descriptions can be directly incorporated into the phase-field model. While for the intermetallic compounds described by the general sublattice model, the internal minimization of sublattice occupancy and solute evolution during microstructure transformation can be simultaneously realized during the numerical simulation. ${ }^{[7]}$ This treatment leads, in general, to a solution superior to the separate solution of the individual problems, and can be regarded to be a "direct" coupling method.

Generally speaking, the phase-field model with finite interface dissipation opens a novel way to directly couple to the CALPHAD thermodynamic databases. The direct coupling to CALPHAD databases can provide precise thermophysical information for the phase-field simulation in an effective way. We believe that the coupling between the phase-field model with finite interface dissipation and the CALPHAD databases may serve as a standard approach for quantitative phase-field simulation of microstructure evolution in materials processes.

\title{
References
}

1. A. Karma, Phase-Field Formulation for Quantitative Modeling of Alloy Solidification, Phys. Rev. Lett., 2001, 87, p 115701

2. I. Steinbach, B. Böttger, J. Eiken, N. Warnken, and S.G. Fries, CALPHAD and Phase-Field Modeling: A Succesful Liaison, J. Phase Equilib. Diffus., 2007, 28(1), p 101

3. L. Zhang, I. Steinbach, and Y. Du, Phase-Field Simulations of Diffusion Couples in the Ni-Al System, Int. J. Mat. Res, 2011, 102(4), p 371

4. J.Z. Zhu, Z.K. Liu, V. Vaithyanathan, and L.Q. Chen, Linking Phase-Field Model to CALPHAD: Application to Precipitate Shape Evolution in Ni-Base Alloys, Scripta Mater, 2002, 46(5), p 401

5. I. Steinbach, L. Zhang, and M. Plapp, Phase-Field Model with Finite Interface Dissipation, Acta Mater., 2012, 60(6-7), p 2689

6. L. Zhang and I. Steinbach, Phase-Field Model With Finite Interface Dissipation: Extension To Multi-Component Multi-Phase Alloys, Acta Mater., 2012, 60(6-7), p 2702

7. L. Zhang, M. Stratmann, Y. Du, B. Sundman, and I. Steinbach, Incorporating The CALPHAD Sublattice Approach of Ordering into the Phase-Field Model with Finite Interface Dissipation, Acta Mater, 2015, 88, p 156

\author{
Lijun Zhang \\ Professor, State Key Lab of Powder Metallurgy \\ Central South University \\ Changsha, Hunan 410083, \\ China \\ E-mail: lijun.zhang@csu.edu.cn \\ Yong Du \\ Associate Editor, Journal of Phase Equilibria and Diffusion \\ Professor, State Key Lab of Powder Metallurgy \\ Central South University, Changsha, Hunan 410083, China \\ E-mail: yong-du@csu.edu.cn; yongducalphad@gmail.com
}

